HNO 2021 · 69:611-614

https://doi.org/10.1007/s00106-021-01084-3

Angenommen: 7. Mai 2021

Online publiziert: 17. Juni 2021

๑) Der/die Autor(en) 2021

Ludger Klimek' $\cdot$ Roland Buhl ${ }^{2}$. Thomas Deitmer ${ }^{3}$ Stefan Plontke ${ }^{4}$.

Wolfgang Wehrmann ${ }^{5} \cdot$ Hans Merk $^{6}$. Johannes Ring ${ }^{7} \cdot$ Sven Becker ${ }^{8}$.

Ärzteverband Deutscher Allergologen (AeDA) - Deutsche Gesellschaft für HalsNasen-Ohren-Heilkunde, Kopf- und Hals-Chirurgie (DGHNO-KHC)

'Zentrum für Rhinologie und Allergologie Wiesbaden, Wiesbaden, Deutschland

${ }^{2}$ Schwerpunkt Pneumologie, III. Medizinische Klinik und Poliklinik, Universitätsmedizin Mainz, Mainz, Deutschland

${ }^{3}$ Deutsche Gesellschaft für Hals-Nasen-Ohren-Heilkunde, Kopf- und Hals-Chirurgie, Bonn, Deutschland

${ }^{4}$ Klinik und Poliklinik für Hals-Nasen-Ohren-Heilkunde, Kopf- und Hals-Chirurgie, Martin-Luther-

Universität Halle-Wittenberg, Halle, Deutschland

${ }^{5}$ Dermatologische Gemeinschaftspraxis Wehrmann, Münster, Deutschland

${ }^{6}$ Abteilung Dermatologie \& Allergologie, RWTH Aachen, Aachen, Deutschland

${ }^{7}$ Haut- und Laserzentrum an der Oper, München, Deutschland

${ }^{8}$ Klinik für Hals-, Nasen- und Ohrenheilkunde, Kopf- und Halschirurgie, Eberhard Karls Universität

Tübingen, Tübingen, Deutschland

\title{
Stellungnahme zum Einsatz von topisch-inhalativem Budesonid bei COVID-19-Infektion
}

\section{Positionspapier des Ärzteverbands Deutscher Allergologen (AeDA) und der Deutschen Gesellschaft für Hals-Nasen- Ohren-Heilkunde, Kopf- und Hals-Chirurgie (DGHNO-KHC)}

Glukokortikosteroide (GKS) gelten als Standardtherapie entzündlicher Erkrankungen der Atemwegsschleimhäute, wie allergische Rhinitis (AR), chronische Rhinosinusitis (CRS) oder Asthma bronchiale (A) $[1,2]$. CRS, AR und A gehören zu den häufigsten entzündlichen Erkrankungen überhaupt, und mit der Chronifizierung sind häufig Epithelschädigungen und Gewebsdestruktionen verbunden, die Virusinfektionen Vorschub leisten können [1, 2].

Die englische Version dieses Beitrags ist unter https://doi.org/10.1007/10.1007/s00106-02101070-9 zu finden.

Die Mitglieder des Ärzteverbands Deutscher Allergologen (AeDA) und der Deutschen Gesellschaft für Hals-Nasen-Ohren-Heilkunde, Kopfund Hals-Chirurgie (DGHNO-KHC) werden am Beitragsende gelistet.
Ein Asthma bronchiale ist eine wichtige Komorbidität von AR und CRS. Verschlechterungen in der Kontrolle von AR und CRS können Asthma-Exazerbationen begünstigen [3-7].

Nachdem in der aktuellen Pandemie durch SARS-CoV-2 („severe acute respiratory syndrome coronavirus type 2 “) zunächst Hinweise publik wurden, dass „Kortison-Präparate“ das Risiko erhöhen, an COVID-19 („coronavirus disease 2019“) zu erkranken, bzw. einen schwereren Verlauf der Erkrankung hervorrufen könnten und hierdurch zahlreiche Patienten mit AR, CRS und Asthma massiv verunsichert wurden, wiesen Positionspapiere der deutschen und europäischen Gesellschaften sehr frühzeitig in der Pandemie auf die Notwendigkeit einer Fortführung der Therapie mit topischen GKS hin $[1,2]$. Demnach sind topische GKS als nasale (nGKS) und inhalative (ICS) Form effektiv in der Behandlung von Schleimhautentzündungen der oberen und unteren Atemwege und stellen die Standardtherapie dieser Erkrankungen dar [8-11].

Es existieren keinerlei Hinweise, dass eine Anwendung von nGKS und ICS ein erhöhtes Risiko für eine SARS-CoV-2Infektion oder einen schwereren Verlauf einer COVID-19-Erkrankung auslösen. Erwachsene und Kinder mit AR, CRS und A sollten daher ihre verordneten nGKS und ICS konsequent und regelmäßig in der individuell verordneten Dosis einnehmen und nicht ohne Rücksprache mit dem behandelnden Arzt ändern oder gar beenden $[1,2]$. Hierbei wiesen wir auch darauf hin, dass eine gute antientzündliche Kontrolle der oberen und unteren Atemwege durch topische GKS nach aktuellem Stand des 
medizinischen Wissens ein guter Schutz vor durch Viren ausgelöste Exazerbationen für diese Patienten darstellt $[1,2]$. Aus heutiger Sicht gibt es genügend Daten, dass Patienten mit chronisch-entzündlichen Atemwegserkrankungen im Rahmen der COVID-19-Pandemie eine leitliniengerechte pharmakologische Behandlung erhalten sollten, die, falls erforderlich, nGKS, ICS und Biologika-Therapien einschließt $[12,13]$. Zu den o.g. topischen Glukokortikosteroiden gehört auch Budesonid.

\section{Studie zur Anwendung von Budesonid bei COVID-19}

In der letzten Ausgabe der Zeitschrift THE LANCET Respiratory Medicine veröffentlichten Ramakrishnan et al. eine hypothesengenerierende Studie über den Einsatz von inhalativem Budesonid im Vergleich zur "Standardtherapie“ bei Patienten mit frühem COVID-19 [14]. Die Autoren führten eine offene, randomisierte kontrollierte Parallelgruppenstudie der Phase II durch, um den Einsatz von inhaliertem Budesonid $(1600 \mu \mathrm{g} / \mathrm{Tag}) \mathrm{mit}$ der Standardtherapie bei Patienten mit in den vergangenen 7 Tagen festgestellter, symptomatischer COVID-19-Erkrankung zu vergleichen [14]. Die Autoren kommen zur Schlussfolgerung, dass diese Behandlungsform die erste kostengünstige und leicht zugängliche therapeutische Intervention bei COVID-19 im Frühstadium sein könnte. Sie diskutieren, dass ihre Daten auch eine potenziell wirksame Behandlung zur Verhinderung langfristiger und anhaltender COVID-19-Symptome darstellen könnte.

\section{Bewertung der Studiendaten}

Die oben dargestellten Aussagen [14] werden unseres Erachtens durch die präsentierten Daten nicht unterstützt. Es handelte sich um eine offene Studie, bei der Patienten und Ärzte über die Art der Therapie informiert waren. Placeboeffekte von ICS bei Asthma bronchiale werden mit $21-46 \%$ beschrieben, insbesondere wenn subjektive Ergebnisparameter als Evaluationskriterien („outcome parameter“) verwendet werden [15].

Die in dieser Studie festgestellten Effekte, einschließlich des primären Endpunkts (COVID-19-bedingte ambulante Vorstellung oder Hospitalisierung) und sekundärer Endpunkte (wie die Zeit bis zur von den Patienten subjektiv empfundenen klinischen Besserung) könnten durch die subjektive Wahrnehmung der betroffenen Patienten und ihrer behandelnden Ärzte beeinflusst worden sein [16].

Objektive Messwerte wie die Sauerstoffsättigung im Blut, die Körpertemperatur, spirometrische Befunde und die quantitative Viruslast mit SARS-CoV-2 wurden als weitere sekundäre Endpunkte in der Studie verwendet, unterschieden sich in ihren Ergebnissen jedoch nicht zwischen den Gruppen. Die Studie war klein und umfasste nur 146 Teilnehmer 73 wurden in die „Standardtherapie “ und 73 in die Budesonid-Gruppe randomisiert.

Ramakrishnan et al. stellen die Hypothese auf, dass die frühzeitige Verabreichung von inhalativem Budesonid die Wahrscheinlichkeit reduziert, dass eine dringende medizinische Versorgung benötigt wird, und die Zeit bis zur Genesung bei einem frühzeitig behandelten COVID-19 verkürzt [14]. In Anbetracht der Evidenz aus dieser und anderen Studien bleibt diese Interpretation der Daten spekulativ.

Der primäre Endpunkt wurde bei $11(15 \%)$ der Kontrollpatienten und 2 (3\%) der Budesonid-Patienten erreicht $(p=0,009)$. Die Zeit bis zur klinischen Besserung war bei den mit Budesonid behandelten Patienten um einen Tag kürzer (7 vs. 8 Tage, $p=0,007)$. Bei $2 \%$ der Budesonid-Patienten trat in den $14 \mathrm{Ta}$ gen nach Studienbeginn Fieber auf, im Vergleich zu $8 \%$ der Kontrollpatienten $(p=0,051)$. Unerwünschte Wirkungen traten bei 5 Patienten $(=7 \%)$ in der Budesonid-Gruppe auf.

Hierzu passen die Zwischenergebnisse einer noch laufenden größeren PhaseIII-Studie an 2617 Patienten, die ebenfalls eine inhalative Budesonid-Therapie bei akuter SARS-CoV-2-Infektion prüft [17]. Hier zeigte sich eine um 3 Tage kürzere Zeit bis zur selbstberichteten kli- nischen Besserung der Symptome, der geringe Effekt auf COVID-19-bedingte Hospitalisierungen oder Todesfälle (Budesonid: 59/692 [8,5\%] vs. 100/968 [10,3\%] Patienten) war allerdings nicht statistisch signifikant.

\section{Diskussion}

Die deutschen Gesellschaften AeDA (Ärzteverband Deutscher Allergologen), DGP (Deutsche Gesellschaft für Pneumologie und Beatmungsmedizin), DGAKI (Deutsche Gesellschaft für Allergologie und klinische Immunologie), DGHNO-KHC (Deutsche Gesellschaft für Hals-Nasen-Ohren-Heilkunde, Kopf- und Hals-Chirurgie) gemeinsam mit internationalen Organisationen wie ARIA (Allergic Rhinitis and its Impact on Asthma Initiative), EAACI (European Academy of Allergy and Clinical Immunology) und GAL ${ }^{2} \mathrm{EN}$ (Global Allergy and Asthma European Network) betonten die Notwendigkeit der Fortführung und des konsequenten Einsatzes von ICS bei erkrankten Patienten bereits zu Beginn der Pandemie [12, 13]. Ähnliche Empfehlungen wurden auch für weitere allergische Erkrankungen während der Pandemie erstellt [18-20].

Die vorliegenden Studienergebnisse zur inhalativen Budesonid-Therapie sprechen dafür, dass bei Beginn einer inhalativen Budesonid-Therapie in hoher Dosis innerhalb weniger Tage nach Beginn einer COVID-19-Erkrankung eine etwas kürzere Symptomdauer erzielt werden kann. Ein höhergradiger klinischer Effekt lässt sich auf Basis der aktuellen Studienlage nicht belegen.

Eine vorsichtige Interpretation dieser Daten ist umso mehr angebracht, als eine aktualisierte Zwischenanalyse von Daten einer größeren Phase-III-Studie, die 2617 Personen mit Risikofaktoren für ungünstige Verläufe von COVID-19 einschloss, nicht so günstige Ergebnisse zeigte [17] - inhaliertes Budesonid verkürzte die Zeit bis zur selbstberichteten Genesung im Median um 3 Tage, erreichte aber nicht den primären Ergebnisparameter (vordefinierter Überlegenheitsschwellenwert für die Wahrscheinlichkeit, dass die Zahlen der COVID-19-Hospitalisierungen/-Todesfälle in der Budesonid- 
Gruppe im Vergleich zur Standardtherapie niedriger waren): Budesonid: 59/692 $(8,5 \%)$ vs. Standardtherapie: $100 / 968$ $(10,3 \%)$ [17].

Neuere Daten geben des Weiteren Hinweise darauf, dass Patienten mit unterschiedlichen Asthma-Endotypen (Typ-2-Asthma vs. Nicht-Typ-2-Asthma) ein unterschiedliches Risikoprofil in Bezug auf die SARS-CoV-2-Infektion, die Entwicklung von COVID-19 und das Fortschreiten zu schweren COVID19-Erkrankungen aufweisen [21].

Effektive Maßnahmen zur Eindämmung der Pandemie sind hingegen Einschränkungen des gesellschaftlichen Lebens, vor allem zum Schutz besonders gefährdeter Patientengruppen und zur Aufrechterhaltung eines funktionierenden Gesundheitssystems. In Übereinstimmung mit RKI (Robert KochInstitut) und WHO (Weltgesundheitsorganisation) empfehlen wir in der aktuellen Pandemie-Situation Präventionsmaßnahmen, wie beispielsweise [22-25]:
- Abstand von mindestens 1,5-2 m zu anderen Personen halten,

- Einhaltung von allgemeinen Hygienemaßnahmen, wie regelmäßige Händedesinfektion/regelmäßiges Händewaschen für mindestens $30 \mathrm{~s}$, Berührung von Schleimhäuten mit den Händen vermeiden,

- Minimierung der sozialen Kontakte,

- Beschränkung von persönlichen Patientenkontakten auf das absolut Notwendige,

- Tragen von persönlicher Schutzkleidung und

- regelmäßige Flächendesinfektion, insbesondere Türklinken etc.,

- möglichst rasche und umfassende Impfkampagnen.

\section{Korrespondenzadresse}

\section{PD Dr. Sven Becker}

Klinik für Hals-, Nasen- und Ohrenheilkunde, Kopf- und Halschirurgie, Eberhard Karls Universität Tübingen

Elfriede-Aulhorn-Str. 5, 72076 Tübingen, Deutschland

Sven.Becker@med.uni-tuebingen.de

Mitglieder des Ärzteverbands Deutscher Allergologen (AeDA) (alphabetisch). Sven Becker, Klinik für Hals-, Nasen- und Ohrenheilkunde, Kopf- und Halschirurgie, Eberhard Karls Universität Tübingen, Tübingen, Deutschland; Ludger Klimek, Zentrum für Rhinologie und Allergologie Wiesbaden, Wiesbaden, Deutschland; Hans Merk, Abteilung Dermatologie \& Allergologie, RWTH Aachen, Deutschland; Johannes Ring, Haut- und Laserzentrum an der Oper, München; Wolfgang Wehrmann, Dermatologische Gemeinschaftspraxis Wehrmann, Münster, Deutschland.

Mitglieder der Deutschen Gesellschaft für HalsNasen-Ohren-Heilkunde, Kopf- und Hals-Chirurgie (DGHNO-KHC) (alphabetisch). Thomas Deitmer, Deutsche Gesellschaft für Hals-NasenOhren-Heilkunde, Kopf- und Hals-Chirurgie, Bonn, Deutschland; Stefan Plontke, Klinik und Poliklinik für Hals-Nasen-Ohren-Heilkunde, Kopf- und Hals-Chirurgie, Martin-Luther-Universität Halle-Wittenberg, Halle, Deutschland.

Hier steht eine Anzeige. 
Funding. Open Access funding enabled and organized by Projekt DEAL.

\section{Einhaltung ethischer Richtlinien}

Interessenkonflikt. L. Klimek, R. Buhl, T. Deitmer, S. Plontke, W. Wehrmann, H. Merk, J. Ring und S. Becker geben an, dass kein Interessenkonflikt besteht.

Für diesen Beitrag wurden von den Autoren keine Studien an Menschen oder Tieren durchgeführt. Für die aufgeführten Studien gelten die jeweils dort angegebenen ethischen Richtlinien.

Open Access. Dieser Artikel wird unter der Creative Commons Namensnennung 4.0 International Lizenz veröffentlicht, welche die Nutzung, Vervielfältigung, Bearbeitung, Verbreitung und Wiedergabe in jeglichem Medium und Format erlaubt, sofern Sie den/die ursprünglichen Autor(en) und die Quelle ordnungsgemäß nennen, einen Link zur Creative Commons Lizenz beifügen und angeben, ob Änderungen vorgenommen wurden.

Die in diesem Artikel enthaltenen Bilder und sonstiges Drittmaterial unterliegen ebenfalls der genannten Creative Commons Lizenz, sofern sich aus der Abbildungslegende nichts anderes ergibt. Sofern das betreffende Material nicht unter der genannten Creative Commons Lizenz steht und die betreffende Handlung nicht nach gesetzlichen Vorschriften erlaubt ist, ist für die oben aufgeführten Weiterverwendungen des Materials die Einwilligung des jeweiligen Rechteinhabers einzuholen.

Weitere Details zur Lizenz entnehmen Sie bitte der Lizenzinformation auf http://creativecommons.org/ licenses/by/4.0/deed.de.

\section{Literatur}

1. Klimek L, Becker S, Buhl R et al (2020) Positionspapier: Empfehlungen zur Behandlung der chronischen Rhinosinusitis während der COVID-19-Pandemie im deutschen Gesundheitssystem - Empfehlungen des Ärzteverbandes Deutscher Allergologen (AeDA) und der Deutschen Gesellschaft für HNO-Heilkunde, Kopfund Halschirurgie (DGHNO-KHC). Laryngorhinootologie 99:356-364. https://doi.org/10.1055/a1164-9696 (Diese Empfehlungen basieren auf dem EAACI Positionspapier "Treatment of chronic RhinoSinusitis with nasal polyps (CRSwNP) in the COVID-19 pandemics-An EAACI Position Paper", Allergy, 2020 und wurden auf die Situation im deutschen Gesundheitswesen angepasst.)

2. Klimek L, Förster-Ruhrmann U, Becker Set al (2020) Stellungnahme zur Anwendung von Glukokortikosteroiden bei entzündlichen Erkrankungen der oberen Atemwege (u.a. allergische Rhinitis/ chronische Rhinosinusitis) während der aktuellen COVID-19-Pandemie - Empfehlungen des Ärzteverbandes Deutscher Allergologen (AeDA), des Deutschen Berufsverbandes der HNO-Ärzte (BVHNO) und der AGs Klinische Immunologie, Allergologie und Umweltmedizin und Rhinologie und Rhinochirurgie der Deutschen Gesellschaft für HNO-Heilkunde, Kopf- und Halschirurgie (DGHNO-
KHC). Laryngorhinootologie 99:280-281. https:// doi.org/10.1055/a-1148-3553

3. Bölke G, Church MK, Bergmann K-C (2019) Comparison of extended intervals and dose reduction of omalizumab for asthma control. Allergo J Int 28:1-4. https://doi.org/10.1007/ s40629-018-0087-6

4. Hamelmann E (2018) Long-acting muscarinic antagonists for the treatment of asthma in children-a new kid in town. Allergo J Int 27:220-227. https://doi.org/10.1007/s40629018-0066-y

5. Ong KY (2019) What's new in the Global Initiative for Asthma 2018 report and beyond. Allergo J Int 28:63-72. https://doi.org/10.1007/s40629-0180079-6

6. Rothe T (2018) A Century of "intrinsic asthma"-a view on the development of phenotyping in asthma in the last 100 years. Allergo $\mathrm{J} \mathrm{Int}$ 27:215-219

7. Sennekamp J (2014) Bronchitis and asthma in the setting of vitamin D deficiency. Allergo J Int 23:113-114. https://doi.org/10.1007/s40629014-0016-2

8. Buhl R, Bals R, Baur X et al (2021) S2k-Leitlinie zur Diagnostik und Therapie von Patienten mit Asthma-Addendum 2020. Pneumologie 75:191-200. https://doi.org/10.1055/a-13520296

9. Buhl R, Bals R, Baur X et al (2017) S2k-Leitlinie zur Diagnostik und Therapie von Patienten mit Asthma. Pneumologie 71:849-919. https://doi. org/10.1055/s-0043-119504

10. Klimek L, Bachert C, Pfaar O et al (2019) ARIA guideline 2019: treatment of allergic rhinitis in the German health system. Allergol Sel 3:22-50. https://doi.org/10.5414/ALX02120E

11. Klimek L, Casper I, Bergmann K-C et al (2020) Therapy of allergic rhinitis in routine care: evidence-based benefit assessment of freely combined use of various active ingredients. Allergo J Int 29:129-138. https://doi.org/10.1007/ s40629-020-00133-7

12. Bousquet J, Akdis C, Jutel M et al (2020) Intranasa corticosteroids in allergic rhinitis in COVID-19 infected patients: an ARIA-EAACI statement. Allergy. https://doi.org/10.1111/all.14302

13. Bousquet J, Jutel M, Akdis CA et al (2020) ARIAEAACl statement on asthma and COVID-19. Allergy 76:689-697. https://doi.org/10.1111/all.14471

14. Ramakrishnan S, Nicolau DV, Langford B et al (2021) Inhaled budesonide in the treatment of early COVID-19 (STOIC): a phase 2, open-label, randomised controlled trial. Lancet Respir Med. https://doi.org/10.1016/S2213-2600(21)00160-0

15. Dutile S, Kaptchuk TJ, Wechsler ME (2014) The placebo effect in asthma. Curr Allergy Asthma Rep 14:456. https://doi.org/10.1007/s11882-0140456-2

16. Frew AJ, Pfaar O (2018) Placebo effects in allergen immunotherapy: an experts' opinion. Allergo J Int 27:162-166. https://doi.org/10.1007/s40629018-0065-z

17. Yu L-M, Bafadhel M, Dorward J et al (2021) Inhaled budesonide for COVID-19 in people at higher risk of adverse outcomes in the community: interim analyses from the PRINCIPLE trial. Cold Spring Harbor Laboratory Press, https://doi.org/10.1101/ 2021.04.10.21254672

18. Jakob T, Klimek L (2020) Allergologie in Zeiten von Covid-19. Allergo J 29:3-3. https://doi.org/10. 1007/s15007-020-2538-x

19. Klimek L, Worm M, Lange L et al (2020) Management von Anaphylaxiegefährdeten Patienten während der Covid-19-Pandemie. Allergo J 29:16-26. https://doi.org/10.1007/s15007-0202618-y

20. Ring J, Beyer K, Biedermann Tet al (2021) Guideline (S2k) on acute therapy and management of anaphylaxis: 2021 update: S2k-Guideline of the German Society for Allergology and Clinical Immunology (DGAKI), the Medical Association of German Allergologists (AeDA), the Society of Pediatric Allergology and Environmental Medicine (GPA), the German Academy of Allergology and Environmental Medicine (DAAU), the German Professional Association of Pediatricians (BVKJ), the Society for Neonatology and Pediatric Intensive Care (GNPI), the German Society of Dermatology (DDG), the Austrian Society for Allergology and Immunology (ÖGAI), the Swiss Society for Allergy and Immunology (SGAI), the German Society of Anaesthesiology and Intensive Care Medicine (DGAI), the German Society of Pharmacology (DGP), the German Respiratory Society (DGP), the patient organization German Allergy and Asthma Association (DAAB), the German Working Group of Anaphylaxis Training and Education (AGATE). Allergo J Int 30:1-25. https://doi.org/10.1007/ s40629-020-00158-y

21. Skevaki C, Karsonova A, Karaulov A et al (2020) Asthma-associated risk for COVID-19 development. J Allergy Clin Immunol 146:1295-1301. https://doi.org/10.1016/j.jaci.2020.09.017

22. Dramburg S, Walter U, Becker S et al (2021) Telemedicine in allergology: practical aspects: a position paper of the Association of German Allergists (AeDA). Allergo J Int. https://doi.org/10. 1007/s40629-021-00167-5

23. Klimek L, Jakob T (2021) Darf's noch etwas mehr (Bürokratie und Regulierungswut) sein? Allergo J 30:3-3. https://doi.org/10.1007/s15007021-4787-8

24. Klimek L, Pfaar O, Worm M (2020) Anwendung von Biologika bei allergischen und Typ-2-entzündlichen Erkrankungen in der aktuellen COVID-19Pandemie - ein Positionspapier von AeDA, DGAKI, GPA, ÖGAI, LGAI, ÖGP, ARIA und EAACI. Use of biologicals in allergic and type- 2 inflammatory diseases in times of the current COVID-19 pandemic-Position paper of AeDA, DGAKI, GPA, ÖGAI, LGAI, ÖGP, ARIA und EAACI. AL 43:255

25. KlimekL, NovakN, HamelmannEetal (2021) Severe allergic reactions after COVID-19 vaccination with the Pfizer/BioNTech vaccine in Great Britain and USA. Allergo J Int 30:51-55. https://doi.org/10. 1007/s40629-020-00160-4 